\title{
Análise Comparativa das Simulações do Modelo de Mesoescala MM5 e Dados Meteorológicos Observados para a Região do Galeão/RJ
}

Comparing Analysis of MM5 Mesoscale Model Simulations and Observating Meteorologic Datasets in Galeão/RJ

Eduardo Barbosa Corrêa ${ }^{1,2}$; Márcio Cataldi ${ }^{3,4}$ \& Luiz Cláudio Gomes Pimentel ${ }^{1,2}$

${ }^{1}$ Departamento de Meteorologia, Instituto de Geociências - IGEO, Centro de Ciências

Matemáticas e da Natureza-CCMN,

Universidade Federal do Rio de Janeiro - UFRJ - ebcorrea@gmail.com

${ }^{2}$ Programa de Engenharia Mecânica-PEM/COPPE/UFRJ

Cidade Universitária - CT - Bloco G - Av. Brigadeiro Trompowsky, s/n, Ilha do Fundão Rio de Janeiro - RJ - 21949-972 - pimentel@acd.ufrj.br

${ }^{3}$ Operador Nacional do Sistema Elétrico Rua da Quitanda, 196/10. andar - Centro, Rio de Janeiro, RJ - 20091-005 - cataldi@ons.org.br

${ }^{4}$ Programa de Engenharia Civil-PEC/COPPE/UFRJ - Cidade Universitária - CT - Bloco G Av. Brigadeiro Trompowsky, s/n, Ilha do Fundão - Rio de Janeiro - RJ - CEP: 21949-972.

Recebido em: 15/03/2006 Aprovado em: 29/03/2006

\section{Resumo}

O principal objetivo desse trabalho é analisar os resultados dos prognósticos obtidos com o modelo de mesoescala MM5, a partir da comparação com dados de observação meteorológica na região do Galeão, avaliando as variáveis direção e intensidade do vento, temperatura e umidade relativa do ar em superfície e em altitude. Foram realizadas simulações para dois períodos de julho de 2003, compreendendo os dias 10 a 12 que apresentaram a passagem de um sistema frontal sobre o estado, e os dias 13 a 15 que se caracterizaram pela presença de um sistema de alta pressão sobre o continente. As direções do campo de vento a $10 \mathrm{~m}$ são razoavelmente bem representadas, embora ocorrendo uma tendência nos prognósticos do modelo em subestimar sua intensidade para os dois períodos analisados. Os melhores resultados ocorrem na faixa de intensidade de 1 a $3 \mathrm{~m} / \mathrm{s}$. As simulações realizadas apresentam maior destreza na reprodução dos perfis verticais de vento e temperatura do que na reprodução dos perfis de umidade relativa do ar e temperatura do ponto de orvalho para os períodos analisados.

Palavras Chave: MM5; ventos; Galeão 


\begin{abstract}
The main objective of this study is to analyze the MM5 Mesoscale Model results comparing with observational datasets in surface and altitude from meteorological station of Galeão International Airport- RIO DE JANEIRO. These datasets are used to evaluate wind direction and intensity, temperature and relative humidity in surface and altitude. Simulations for two periods of July 2003 were carried through days 10-12 when a frontal system cross Rio de Janeiro, and days 13-15, characterized by presence of high-pressure system over continent. The $10 \mathrm{~m}$ wind direction are well simulated, but results had demonstrated a trend of model to underestimate wind intensity. The best model results are in $1-3 \mathrm{~m} / \mathrm{s}$ intensity band. The simulations got better results in reproducing wind and temperature vertical profiles than dewpoint temperature profiles and relative humidity for the analyzed periods.
\end{abstract}

Keyword: MM5; wind; Galeão

\title{
1 Introdução
}

A região metropolitana do Rio de Janeiro apresenta, além de uma alta densidade demográfica, um elevado grau de industrialização. Estas características apresentam como conseqüência uma grande concentração de fontes de emissão de poluentes que, aliada as peculiares condições meteorológicas e topográficas da região, conduzem a um cenário de degradação atmosférica.

As situações de subsidência, inversão térmica, calmaria, baixa umidade relativa do ar e névoa seca são condições altamente propícias à concentração de poluentes na atmosfera. Em contrapartida, com a intensificação de situações de instabilidade atmosférica, ocorre uma diminuição da concentração de poluentes. Os efeitos desses fenômenos atmosféricos sobre a concentração de contaminantes ocorrem principalmente no interior da Camada Limite Atmosférica (CLA).

Atualmente os modelos numéricos s ao importantes ferramentas para prognosticar os fenômenos atmosféricos que ocorrem em todas as escalas meteorológicas. Entretanto, uma das limitações dessas ferramentas é a ampla utilização de parametrizações na modelagem dos processos da CLA. Considerando que a maioria dessas parametrizações foi desenvolvida a partir de medidas realizadas principalmente nos Estados Unidos e Europa, torna-se necessário o estudo da influência desses modelos na simulação de parâmetros meteorológicos em outras regiões. 
Nos últimos anos vários autores têm se dedicado ao estudo de parametrizações de CLA, presentes principalmente nos modelos de mesoescala, como o PSU/NCAR Mesoscale Modeling System - MM5 (Grell et alii, 1995) utilizado no presente trabalho. Costa e Souza (2002) realizaram simulações com o objetivo de testar a parametrização MRF em prognósticos para o campo de vento a $10 \mathrm{~m}$, verificando uma tendência do modelo MM5 em subestimar essa variável. Este resultado também foi encontrado por Atmet (2002). Arellano et alii, (2002) efetuou simulações utilizando a parametrização MRF verificando que os campos de umidade no interior da CLA não foram corretamente prognosticados. Bright e Mullen (2002) verificaram que o modelo utilizando a parametrização MRF não obteve sucesso em calcular parâmetros relacionados a convecção como o CAPE e CINE.

O objetivo do estudo é avaliar a destreza do modelo de mesoescala MM5, utilizando a parametrização MRF, no prognóstico dos campos de vento, temperatura e umidade relativa do ar em diversos níveis da atmosfera da Região Metropolitana do Rio de Janeiro (RMRJ). Os prognósticos foram comparados com os dados de superfície e altitude obtidos na estação meteorológica doAeroporto Internacional do Galeão - RJ (SBGL), para o período de julho de 2003.

\section{Descrição do Modelo}

A descrição do modelo MM5 apresentada neste trabalho segue Dudhia et ali (2004) e Grell et alii. (1995). O Sistema MM5 é um modelo de simulação numérica desenvolvido no final da década de 70 pela Penn State University em conjunto com o National Center for Atmospheric Research (NCAR). Atualmente se encontra na $5^{a}$. geração e possui como características principais a capacidade de múltiplos aninhamentos de grade, dinâmica não hidrostática e assimilação de dados em 4 dimensões, além de várias parametrizações físicas e portabilidade em diversas plataformas computacionais, incluindo o sistema LINUX. O MM5 utiliza um sistema de coordenadas sigma que segue a topografia do terreno e resolve as equações de Navier-Stokes em três dimensões, a equação da continuidade, a $1^{\mathrm{a}}$. Lei da Termodinâmica e a equação de transferência radiativa.

\section{Metodologia}

Nesta seção será descrita a metodologia utilizada no trabalho para desenvolvermos as simulações das condições atmosféricas e comparações com dados observados, nos períodos de 10-12 e 13-15 de julho/2003, utilizando 
Análise Comparativa das Simulações do Modelo de Mesoescala MM5 e Dados Meteorológicos Observados para a Região do Galeão/RJ

Eduardo Barbosa Corrêa; Márcio Cataldi \& Luiz Cláudio Gomes Pimentel

\begin{tabular}{|c|c|c|c|}
\hline \multirow{2}{*}{ Domínios } & \multicolumn{3}{|c|}{ Características } \\
\cline { 2 - 4 } & Pontos em x e y & Resolução Espacial(km) & Topografia USGS(km) \\
\hline 1 & $25 \times 30$ & 37 & 19 \\
\hline 2 & $34 \times 37$ & 12.3 & 9 \\
\hline 3 & $34 \times 34$ & 4.1 & 1 \\
\hline
\end{tabular}

Tabela 1 Esquemas de grades utilizadas nas simulações com o modelo MM5

o modelo de mesoescala MM5. Nas simulações desenvolvidas nesse estudo foram utilizadas três grades, centralizadas com base na latitude/longitude do SBGL, considerando diferentes resoluções espaciais e topográficas, que são descritas na Tabela 1. Em todos os domínios foram utilizados dados de topografia e elevação United State Geological Survey (USGS).

Os critérios utilizados para montagem dos esquemas de aninhamento de grade no modelo MM5 foram a relação da resolução espacial de 3:1, necessária para que a interação bidirecional entre os domínios seja realizada pelo MM5. Esse critério de decaimento de grade também foi utilizado para definir a relação da grade de mais baixa resolução do MM5 (Domínio 1) com a grade do NCEP. Essa iniciativa foi no sentido de amenizar o impacto da assimilação realizada pela grade de mais baixa resolução do MM5 (com resolução de 37 $\mathrm{km}$ ), com a grade do modelo global do NCEP (com resolução de $1^{\circ} \sim 111 \mathrm{~km}$ ), como já demonstrado por Corrêa et alii. (2004). Foram utilizados 55 níveis verticais em todos os domínios, sendo aproximadamente 26 níveis até $850 \mathrm{hPa}$. O modelo foi inicializado com dados meteorológicos do modelo global AVN (atualmente chamado GFS - Global Forecast System model) do National Centers for Environment Prediction (NCEP) com resolução espacial de 1 grau e resolução temporal de 3 horas. A análise foi realizada utilizando o campo de vento (direção e intensidade) a $10 \mathrm{~m}$ e as sondagens simuladas no modelo MM5 e comparados, respectivamente, com dados observados do código Metar e dados da radiossondagens da atmosfera, obtidos da estação meteorológica do aeroporto internacional do Galeão (SBGL), localizado na latitude de 22,81o e longitude de 43,25o . Na modelagem foram utilizadas as seguintes opções físicas: a parametrização de cumulus proposta por Grell (Grell, 1993), o modelo de microfísica de nuvens e radiação atmosférica (Dudhia, 1989), o modelo de solo com 5 camadas proposto por Dudhia (Dudhia,1996) e a parametrização de Camada Limite Atmosférica MRF, baseada no trabalho de Troen \& Mahrt (1986) e Hong \& Pan (1996). Essa parametrização utiliza uma combinação de fechamento local com a Teoria $\mathrm{K}$, com um fechamento não local que inclui um termo de contra-gradiente apropriado para a simulação do regime de camada limite convectiva. 
Para avaliação das simulações foram gerados gráficos do tipo "rosas de vento" para todos os períodos escolhidos, bem como sondagens nos horários das $00 \mathrm{Z}$ e $12 \mathrm{Z}$ que foram comparados com os dados observados do Aeroporto do Galeão (SBGL). Além disso, foram gerados índices estatísticos para avaliar o desempenho do modelo no que diz respeito à estimativa dos dados observados. Os índices escolhidos para este trabalho são descritos na Tabela 2, onde os termos $X_{P}, X_{O}$, e $\sigma_{O}$ representam os valores prognosticados, observados, e os desvios padrões dessas variáveis. Os parâmetros $\mu_{P}$ e $\mu_{o}$ são as médias dos valores simulados e observados e $n$ é o número de elementos utilizado no cálculo.

\begin{tabular}{|c|c|}
\hline $\begin{array}{l}\text { Coeficiente de Correlação } \\
\text { (COR) }\end{array}$ & $\begin{array}{c}\operatorname{COR}=\frac{\operatorname{Cov}\left(X_{p}, X_{O}\right)}{\sigma_{p}, \sigma_{O}} \\
\operatorname{Cov}\left(X_{p}, X_{O}\right)=\frac{1}{n} \sum_{i-1}^{n}\left(X_{p_{i}}-\mu_{p}\right)\left(X_{a}-\mu_{O}\right)\end{array}$ \\
\hline $\begin{array}{l}\text { Erro Quadrático Médio } \\
\text { Absoluto (EQMA) }\end{array}$ & $E Q M A=\sqrt{\sqrt{\left(X_{P}-X_{O}\right)^{2}}}$ \\
\hline $\begin{array}{l}\text { Erro Quadrático Médio } \\
\text { Relativo (EQMR) }\end{array}$ & $E Q M R=\overline{\left(\frac{E Q M A}{X_{0}} * 100\right)}$ \\
\hline Fator de 2 & $F a 2=\frac{\overline{X_{P}}}{\overline{X_{O}}}$ \\
\hline Desvio Fracional & $F b=\frac{2\left(\overline{X_{O}}-\overline{X_{p}}\right)}{\left(\overline{X_{O}}+\overline{X_{p}}\right)}$ \\
\hline Desvio Fracional Padrão & $F s=\frac{2\left(\sigma_{0}-\sigma_{p}\right)}{\sigma_{0}+\sigma_{p}}$ \\
\hline
\end{tabular}

Tabela 2 Índices estatísticos utilizados

\section{Resultados}

Nesta seção serão apresentados os principais resultados obtidos neste trabalho, englobando as simulações realizadas com o modelo MM5 para o campo de vento a $10 \mathrm{~m}$ e o perfil vertical do vento, temperatura e umidade.

\subsection{Resultados do período de 10 a 12 de julho 2003}

Este período foi caracterizado pela passagem de um sistema frontal sobre o estado do Rio de Janeiro no dia 11. Nesse dia, como apresentado na 
figura 1, o METAR registrou as freqüências principais de SW e W, o que já era esperado em virtude da passagem do Sistema Frontal pelo estado. As simulações realizadas com o modelo reproduziram bem estas freqüências, apesar de subestimar a intensidade do vento. Foi registrado um alto índice de calmaria, inexistente nos dados observados.

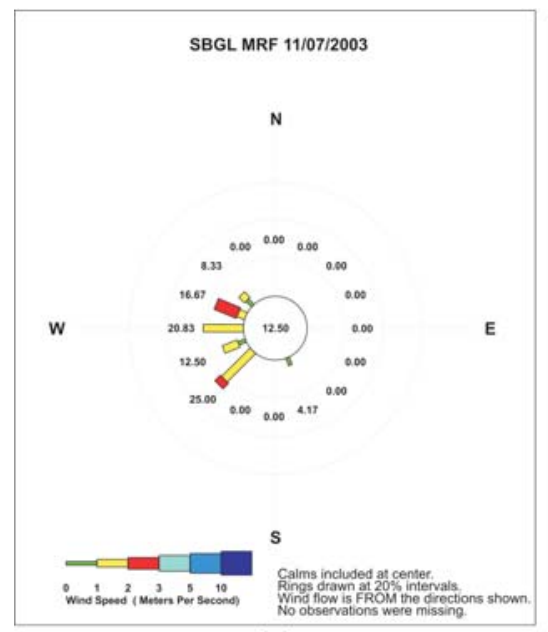

(a)

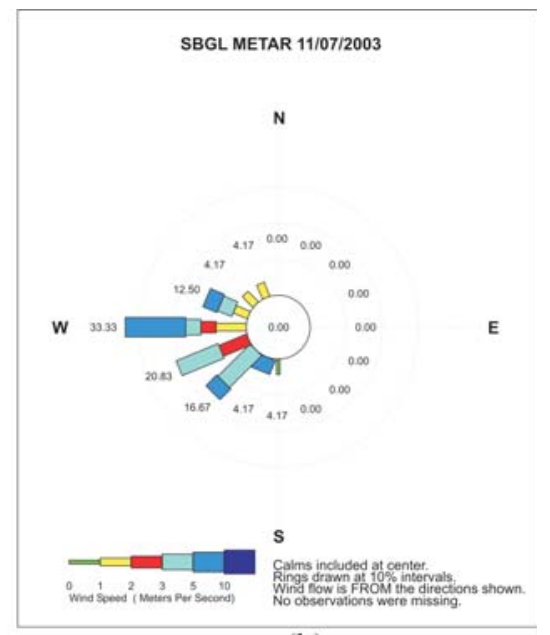

(b)

Figura 1 Rosa dos ventos referente ao dia 11 de julho de 2003.(a) MM5 ; (b) dados observados do METAR (Fonte www.redemet.aer.mil.br)

No dia 12 (Figura 2) as freqüências de vento predominantes foram do quadrante NW, com uma pequena ocorrência em SW-W. As simulações realizadas com o modelo reproduziram as freqüências de NW, subestimando de forma significativa a intensidade do vento, além de registrarem uma freqüência de S com ventos bastante intensos, o que os dados observados não registraram.

A seguir analisaremos as sondagens geradas as $00 \mathrm{Z}$ e $12 \mathrm{Z}$ pelo modelo comparando-as com os resultados das radiossondagens do SBGL, no intuito de verificar o comportamento do vento nos diferentes níveis verticais das simulações realizadas com o modelo MM5. A comparação se iniciará sempre as 00Z do segundo dia de simulação.

No dia 11 as $00 Z$ (Figura 3) a radiossondagem do Galeão registrou o vento em superfície de SW, condizente com a chegada do Sistema Frontal, enquanto as simulações realizadas com o modelo reproduziram o vento ainda de SE, não acusando o giro do vento. Nos níveis médios e altos o vento foi 


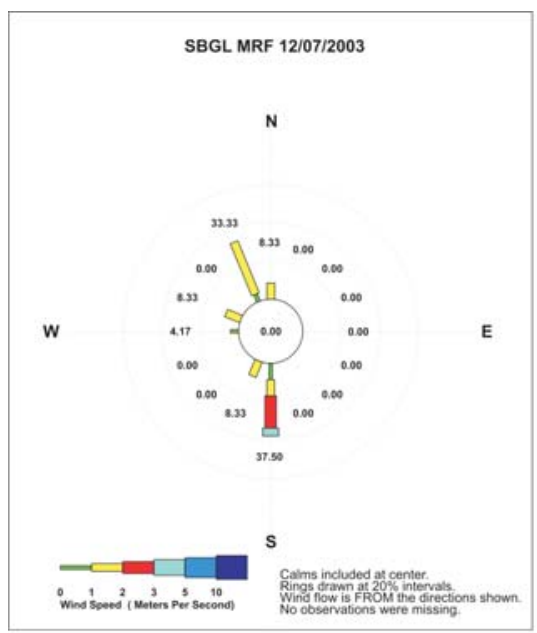

(a)

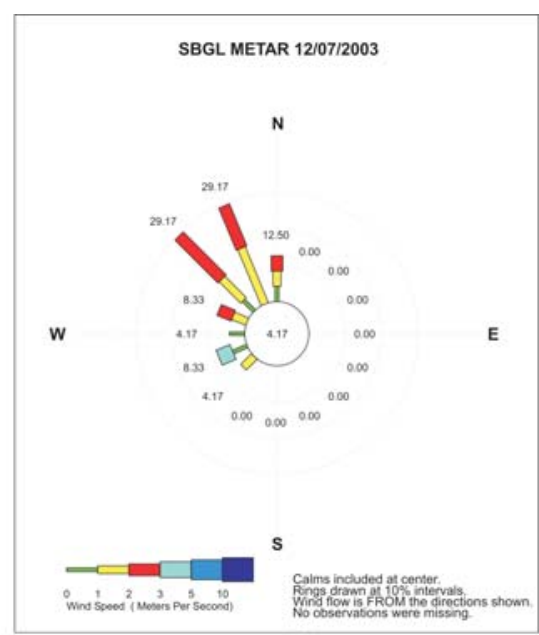

(b)

Figura 2 Rosa dos ventos referente ao dia 12 de julho de 2003.(a) MM5 ; (b) dados observados do METAR (Fonte www.redemet.aer.mil.br)

corretamente reproduzido pelo modelo, bem como a temperatura e a umidade na superfície. Acima do nível de $600 \mathrm{hPa}$ as simulações não registraram satisfatoriamente o perfil vertical termodinâmico da atmosfera, evidenciado pelo distanciamento das linhas de T e Td, ocorrendo então nesses casos uma superestimativa da umidade por parte das simulações realizadas pelo modelo. Entretanto, ocorreu uma inversão no nível entre $900 \mathrm{hPa}$ e $800 \mathrm{hPa}$ que foi registrado corretamente nos prognósticos do modelo MM5.

No dia 11 as $12 Z$ (Figura 4) a radiossondagem registrou o vento em superfície permanecendo de SW, girando para NW em altitude. Os resultados obtidos com o modelo MM5 representaram esse comportamento, descrevendo corretamente o perfil termodinâmico vertical da atmosfera até os níveis de 700 $\mathrm{hPa}$, onde passaram a superestimar os valores de umidade relativa.

No dia 12 as $12 Z$ (Figura 5) foi registrado nas camadas próximas a superfície, vento de NW e SE, o que não foi reproduzido nas simulações do modelo MM5, que apresentaram vento apenas na direção SE. A temperatura e umidade em superfície foram bem representadas, apesar das simulações do modelo representarem uma camada superficial menos úmida do que a radiossondagem. A partir do nível de $500 \mathrm{hPa}$, ocorreu uma significativa diminuição de umidade da atmosfera, que foi simulada com uma ligeira subestimativa que se estendeu até o nível de $300 \mathrm{hPa}$, onde os resultados obtidos nas simulações do modelo MM5 passaram então a superestimar os valores de umidade. Os ventos nesses níveis foram corretamente simulados. 

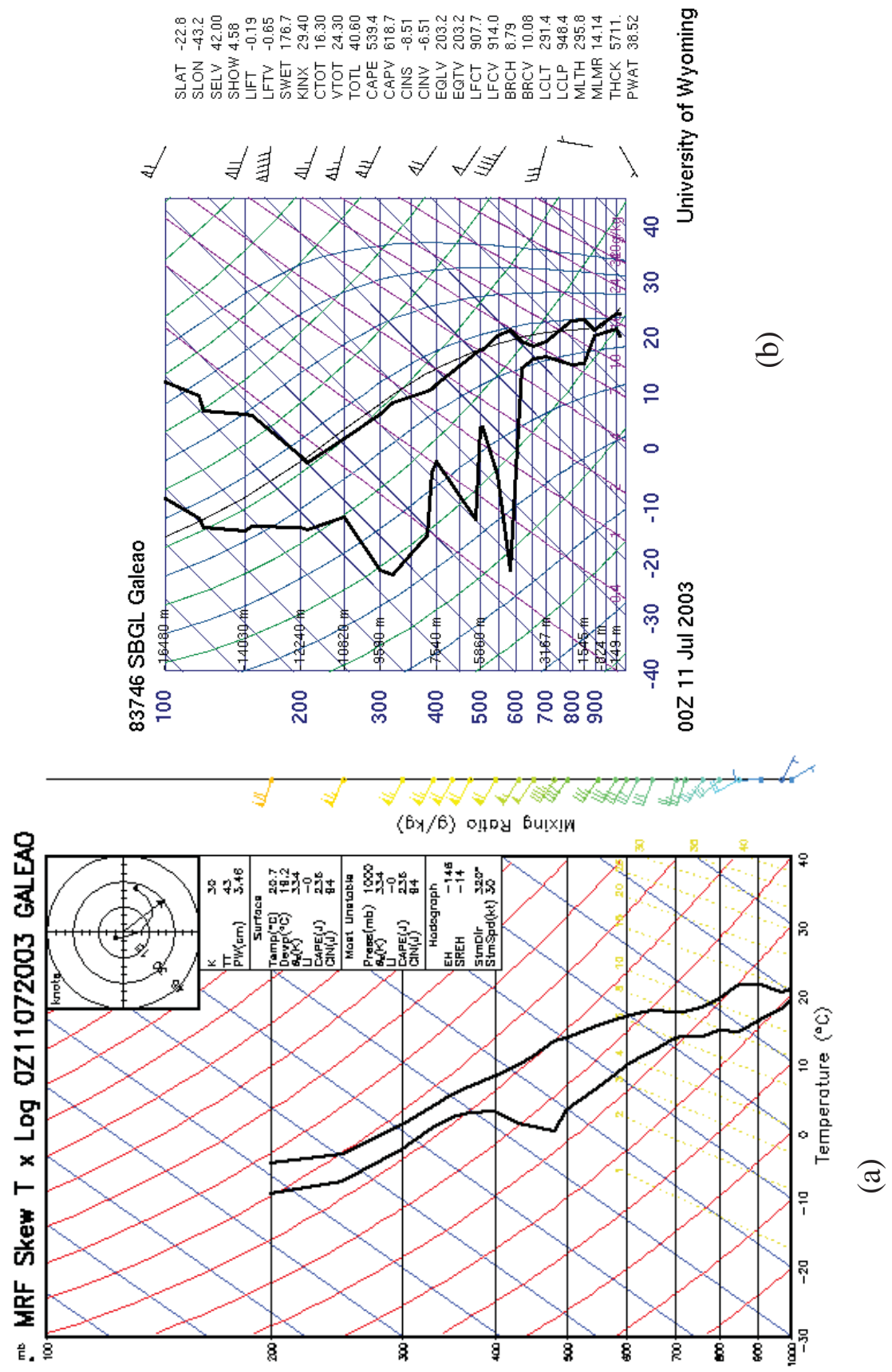

Figura 3 Sondagem referente ao dia 11 de julho de 2003 as 00Z.(a) MM5 ; (b) SBGL (Fonte: weather.uwyo.edu/upperair/samer.html) 

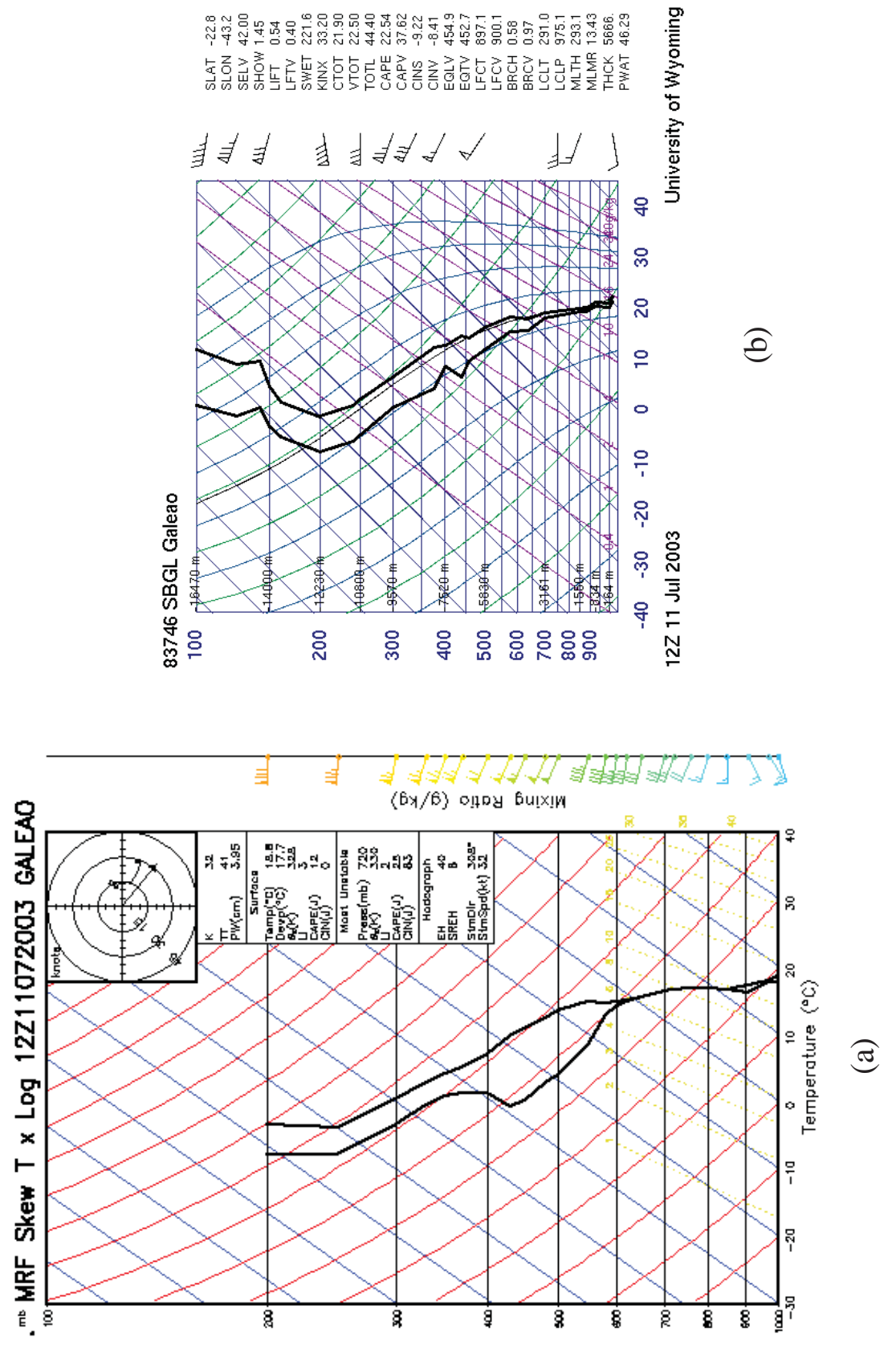

Figura 4 Sondagem referente ao dia 11 de julho de 2003 as 12Z.(a) MM5 ; (b) SBGL (Fonte: weather.uwyo.edu/upperair/samer.html) 


\section{Análise Comparativa das Simulações do Modelo de Mesoescala MM5 e Dados Meteorológicos Observados para a Região do Galeão/RJ}

Eduardo Barbosa Corrêa; Márcio Cataldi \& Luiz Cláudio Gomes Pimentel
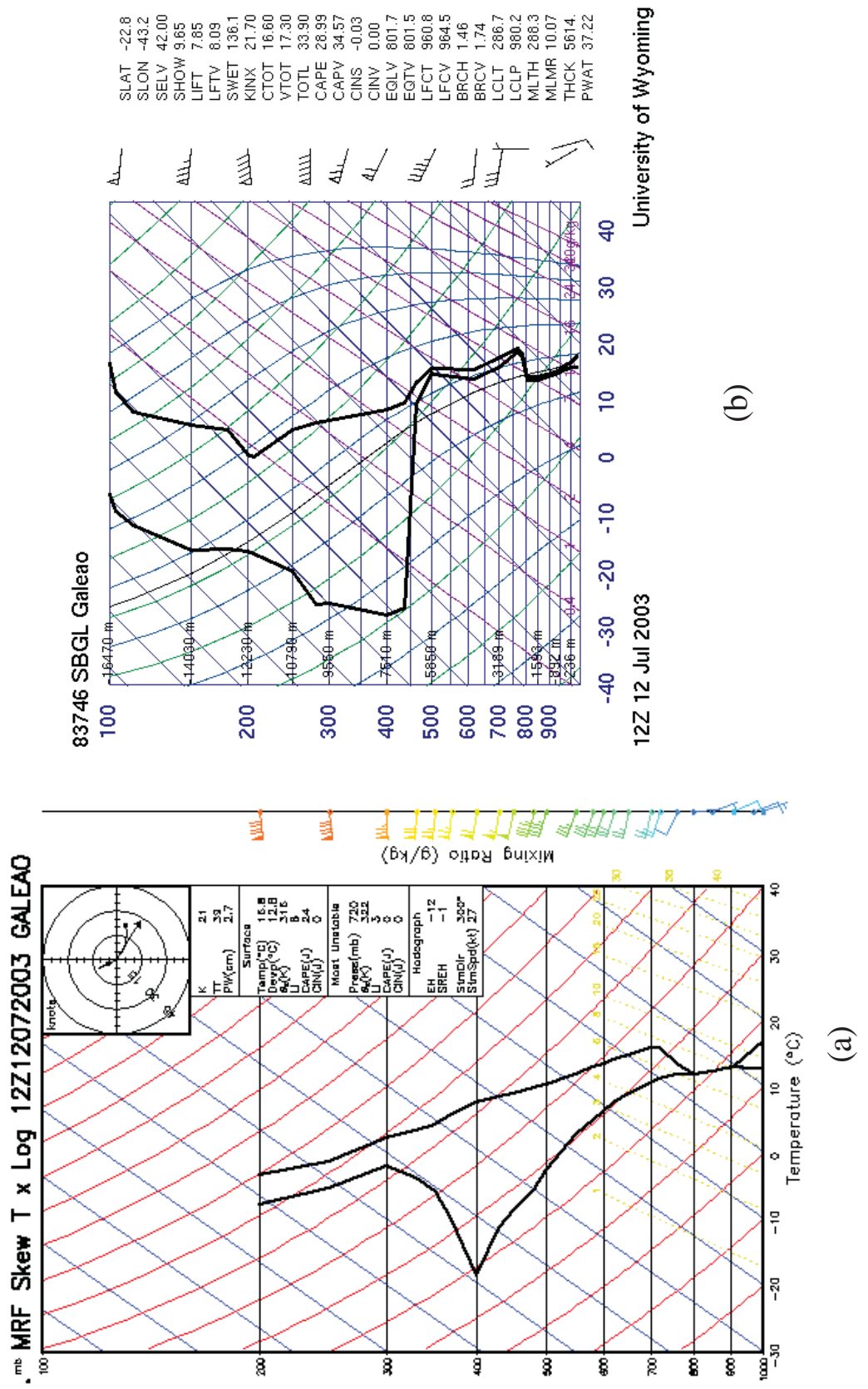

Figura 5 Sondagem referente ao dia 12 de julho de 2003 as 12Z.(a) MM5 ; (b) SBGL (Fonte: weather.uwyo.edu/upperair/samer.html) 
A Tabela 3 mostra os índices estatísticos calculados para o período de 10 a 12 de julho, separados por faixas de intensidade. Vale ressaltar que a $1^{\mathrm{a}}$. faixa apresenta poucos elementos para avaliação. Nas outras faixas, avaliandose os resultados dos índices, podemos observar que quanto maior a intensidade observada dos ventos, maior a propensão ao erro dos resultados obtidos com o modelo, ou seja, maior é a sua tendência em subestimar os valores observados.

Este fato fica evidente principalmente nos valores crescentes apresentados pelo índice EQMR. Os índices Fb, Fa2 e Fs também indicam uma maior tendência a subestimar a intensidade do vento conforme sua intensidade aumenta. Os prognósticos do modelo MM5 aparentemente apresentaram melhor desempenho na faixa de 1 a $3 \mathrm{~m} / \mathrm{s}$.

\begin{tabular}{|c|c|c|c|c|c|c|c|}
\hline $\begin{array}{c}\text { Qtd } \\
\text { elementos }\end{array}$ & $\begin{array}{c}\text { Faixas de } \\
\text { Intensidade (m/s) }\end{array}$ & EQMA(m/s) & EQMR (\%) & COR & FA2 & FB & FS \\
\hline \hline $2(0)^{*}$ & $0 \leq 1 \leq 1$ & 0.7 & 74 & -1 & 1.7 & -0.5 & -1.1 \\
\hline 38 & $1<1 \leq 3$ & 1.0 & 44 & 0.3 & 0.7 & 0.4 & -0.7 \\
\hline 24 & $3<1 \leq 5$ & 2.2 & 54 & -0.1 & 0.5 & 0.8 & -0.4 \\
\hline 8 & $5<1$ & 4.3 & 69 & -0.3 & 0.3 & 1.1 & 0.2 \\
\hline 72 & 3 dias & 1.7 & 51 & 0.2 & 0.5 & 0.6 & 0.5 \\
\hline \hline
\end{tabular}

Tabela 3 Indicadores estatísticos por faixas de intensidade de vento para o período de 10 a 12 de julho de 2003. Simulações com MM5

\subsection{Resultados do período de 13 a 15 de julho 2003}

Nesse período o estado do Rio de Janeiro estava sob influência de um sistema de alta pressão. No dia 14 (Figura 6) os dados observados registraram como $1^{\text {a }}$ freqüência $E$ e $2^{\mathrm{a}} \mathrm{NE}$, que foram corretamente representadas pelas simulações realizadas pelo modelo MM5, embora a ordem tenha sido invertida, simulando como $1^{\text {a }}$ freqüência NE e $2^{\mathrm{a}}$ freqüência de $\mathrm{E}$. 


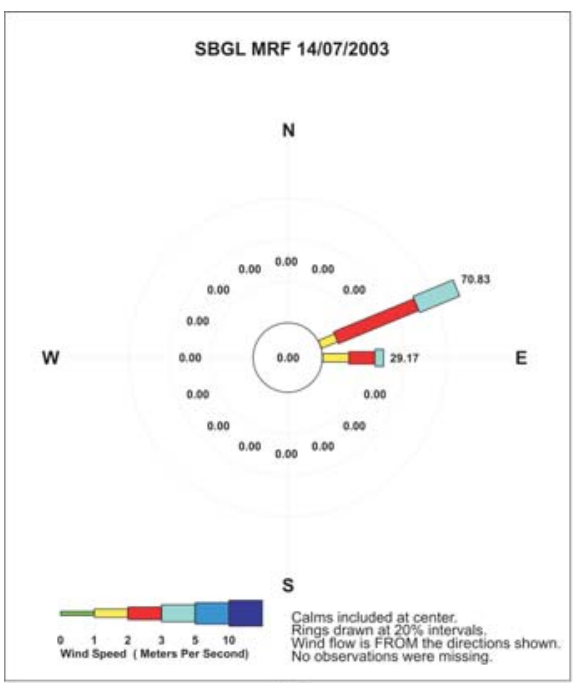

(a)

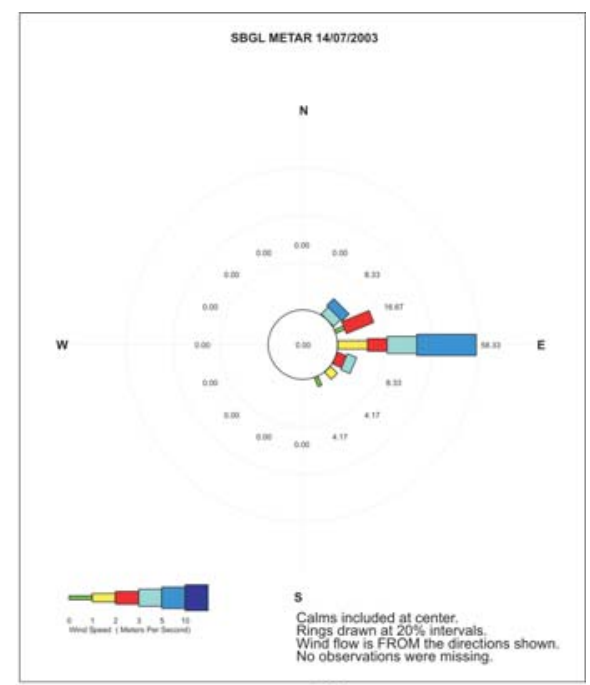

(b)

Figura 6 Rosa dos ventos referente ao dia 14 de julho de 2003.(a) MM5 ; (b) dados observados do METAR (Fonte www.redemet.aer.mil.br)

No dia 15 (Figura 7) a distribuição das freqüências se manteve a mesma do dia anterior, e as simulações também mantiveram suas distribuições. As simulações realizadas com o modelo registraram de forma mais satisfatória a distribuição do vento, quando comparada com os resultados do dia 14.

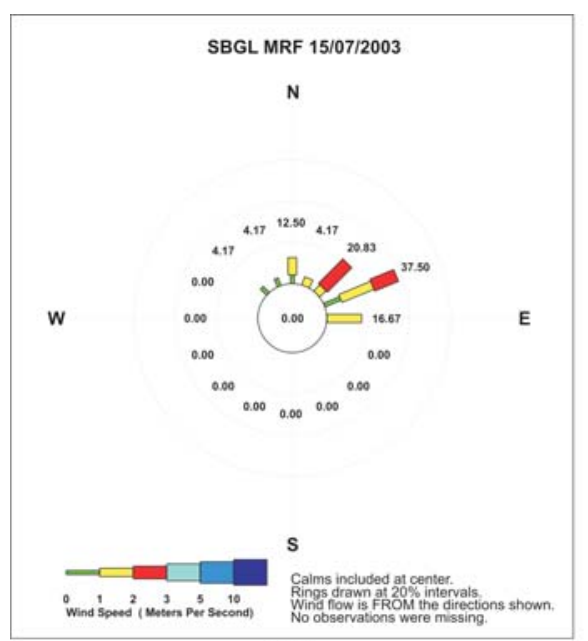

(a)

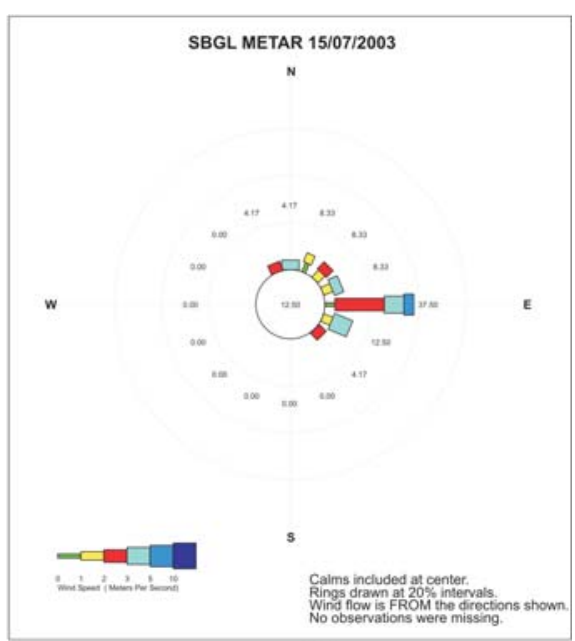

(b)

Figura 7 Rosa dos ventos referente ao dia 15 de julho de 2003.(a) MM5 ; (b) dados observados do METAR (Fonte www.redemet.aer.mil.br) 
No dia 14 as 00Z (Figura 8) a camada de superfície encontrava-se ligeiramente mais úmida, o que foi representado com acurácia pelas simulações realizadas pelo modelo MM5, principalmente na região próxima ao solo. Em altitude os perfis de T e Td mantiveram o mesmo padrão, sendo novamente bem representados pelas simulações realizadas com modelo MM5, assim como os campos de vento, que nas primeiras camadas da atmosfera encontravamse de $\mathrm{E}$, girando para $\mathrm{W}$ em altitude.

No dia 14 as $12 Z$ (Figura 9) o vento na camada superficial encontra-se de $\mathrm{E}$ e ocorre um giro para $\mathrm{W}$ em $700 \mathrm{hPa}$. As simulações realizadas com o modelo registraram corretamente este comportamento. Quanto aos perfis de $\mathrm{T}$ e Td, nos primeiros níveis da atmosfera, as simulações reproduziram uma atmosfera mais úmida do que o observado, sendo esse comportamento invertido em altitude.

No dia 15 as $00 Z$ (Figura 10) o vento registrado próximo a superfície pela radiossondagem estava de $\mathrm{E}$ a $\mathrm{SE}$ e o modelo simulou o vento de $\mathrm{E}$. Quanto aos perfis de T e Td as simulações realizadas com o modelo MM5 reproduziram de modo satisfatório as variações verticais dessas variáveis, exceto nos níveis acima de $400 \mathrm{hPa}$ onde os valores de umidade relativa foram superestimados pelo modelo.

No dia 15 as $12 Z$ (Figura 11) a radiossondagem registrou o vento de $\mathrm{N}$ a NE, girando em altitude para SW, o que foi aparentemente bem representado pelas simulações realizadas com o modelo MM5. Foi simulada uma atmosfera mais úmida, reproduzindo satisfatoriamente o comportamento observado na radiossondagem. No restante do perfil vertical da atmosfera as simulações realizadas com o modelo MM5 apresentaram comportamento condizente com os valores observados.

No dia 16 as $00 Z$ (Figura 12) a radiossondagem registrou vento de SE na camada superficial, enquanto as simulações realizadas com o modelo MM5 apresentaram ventos de NE. Os perfis de T e Td foram simulados corretamente para este horário. 


\section{Análise Comparativa das Simulações do Modelo de Mesoescala MM5 e Dados Meteorológicos Observados para a Região do Galeão/RJ}

Eduardo Barbosa Corrêa; Márcio Cataldi \& Luiz Cláudio Gomes Pimentel
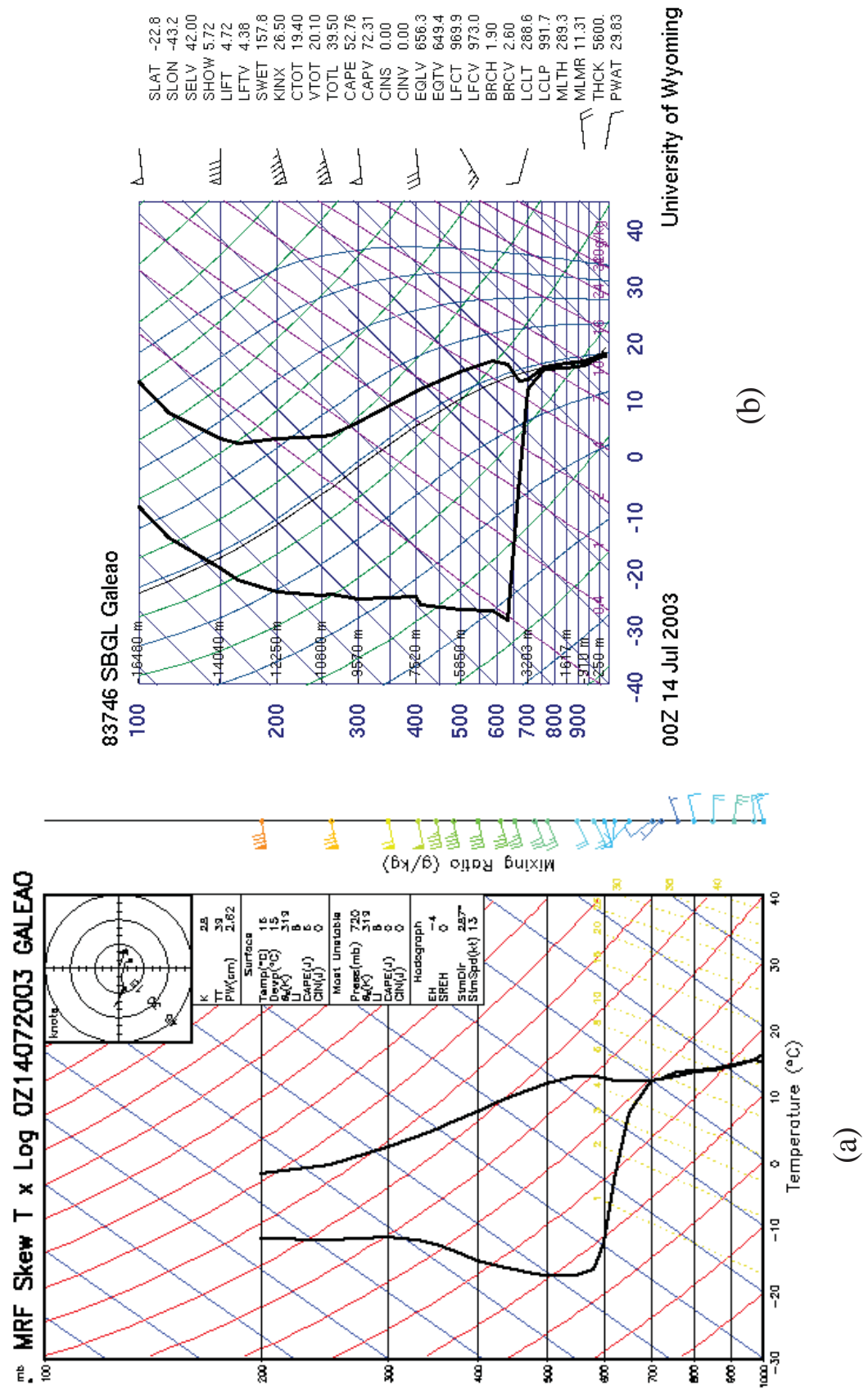

Figura 8 Sondagem referente ao dia 14 de julho de 2003 as 00Z.(a) MM5 ; (b) SBGL (Fonte: weather.uwyo.edu/upperair/samer.html) 

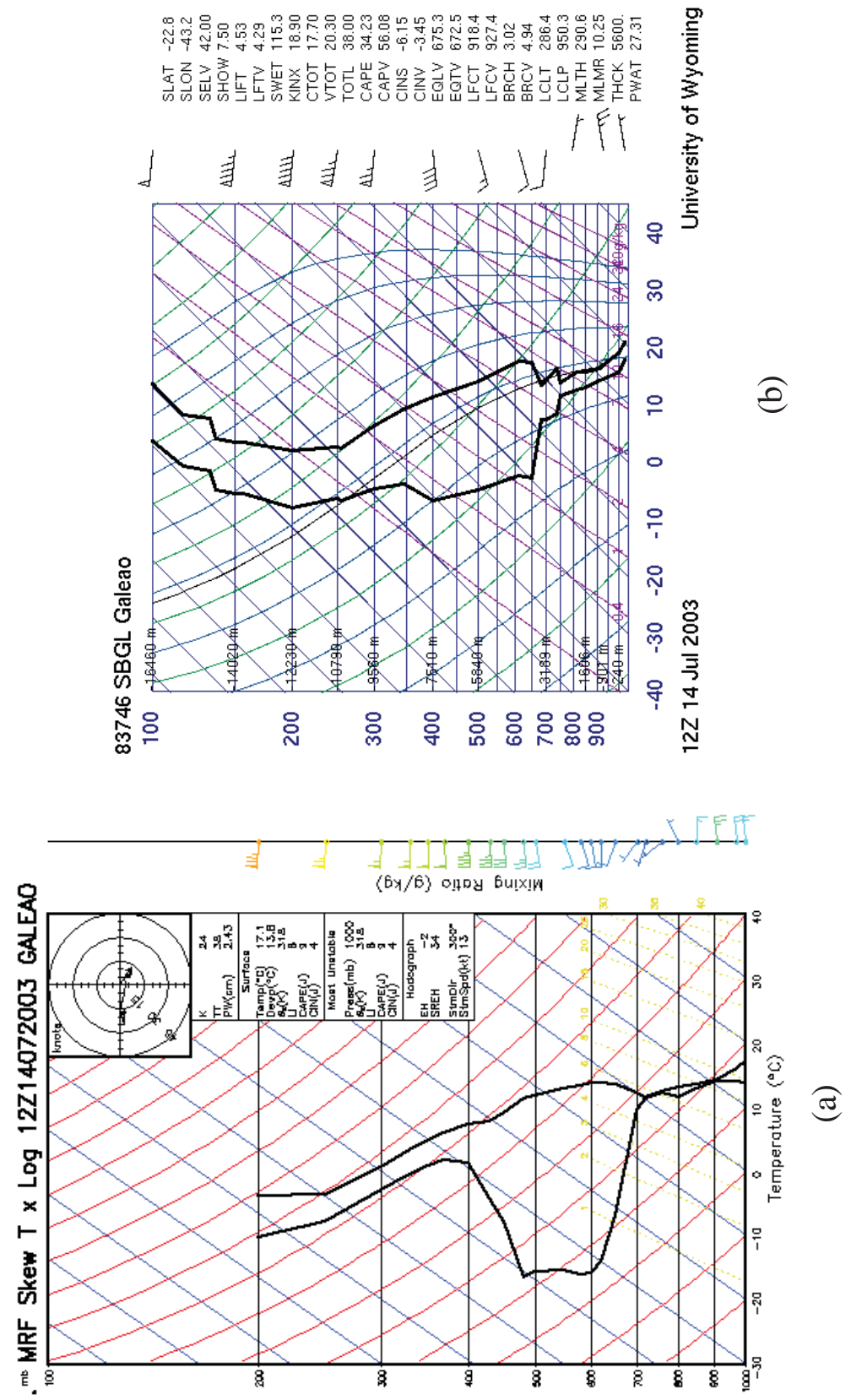

Figura 9 Sondagem referente ao dia 14 de julho de 2003 as 12Z.(a) MM5 ; (b) SBGL (Fonte: weather.uwyo.edu/upperair/samer.html) 


\section{Análise Comparativa das Simulações do Modelo de Mesoescala MM5 e Dados Meteorológicos}

Observados para a Região do Galeão/RJ

Eduardo Barbosa Corrêa; Márcio Cataldi \& Luiz Cláudio Gomes Pimentel
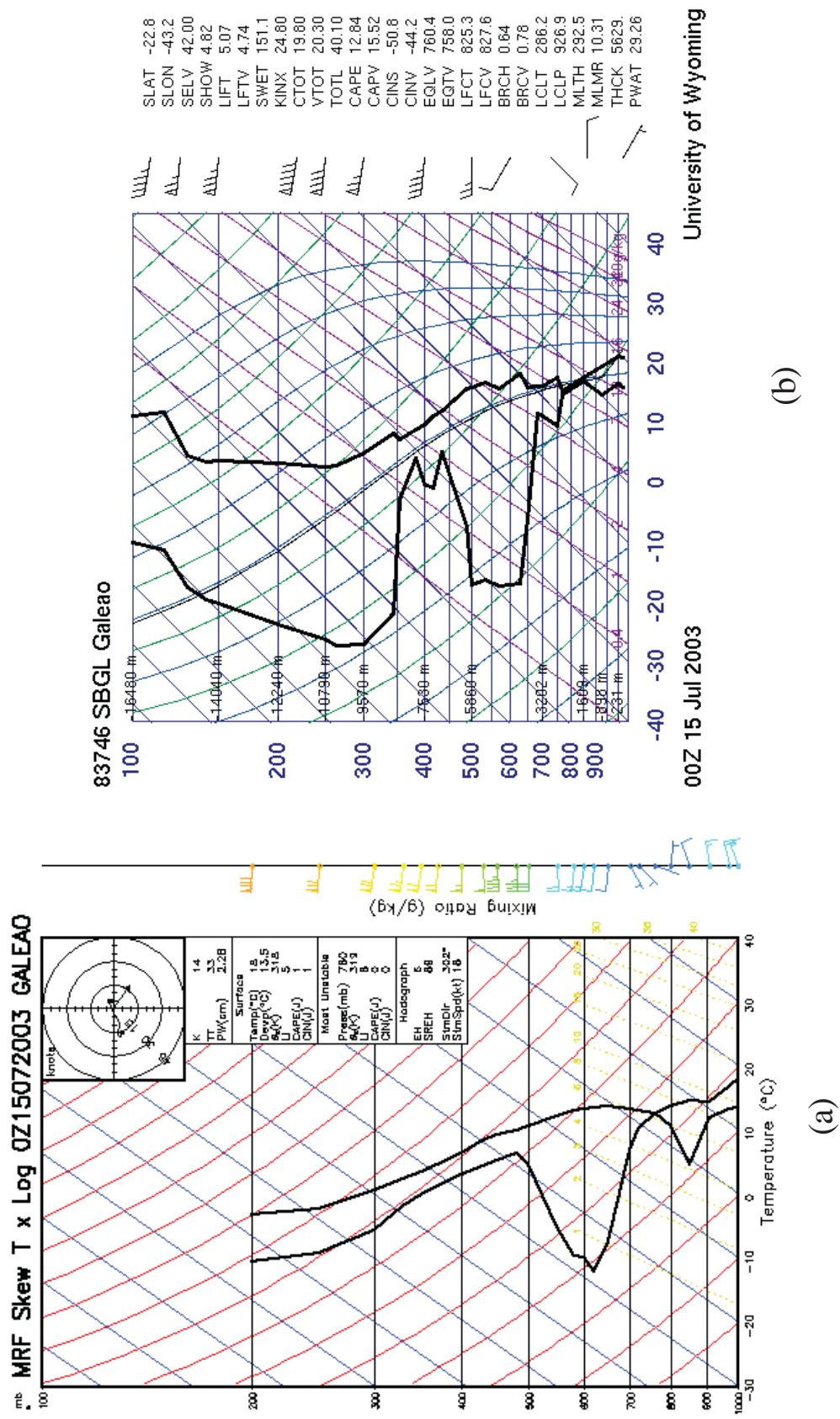

Figura 10 Sondagem referente ao dia 15 de julho de 2003 as 00Z.(a) MM5 ; (c) SBGL (Fonte: weather.uwyo.edu/upperair/samer.html) 

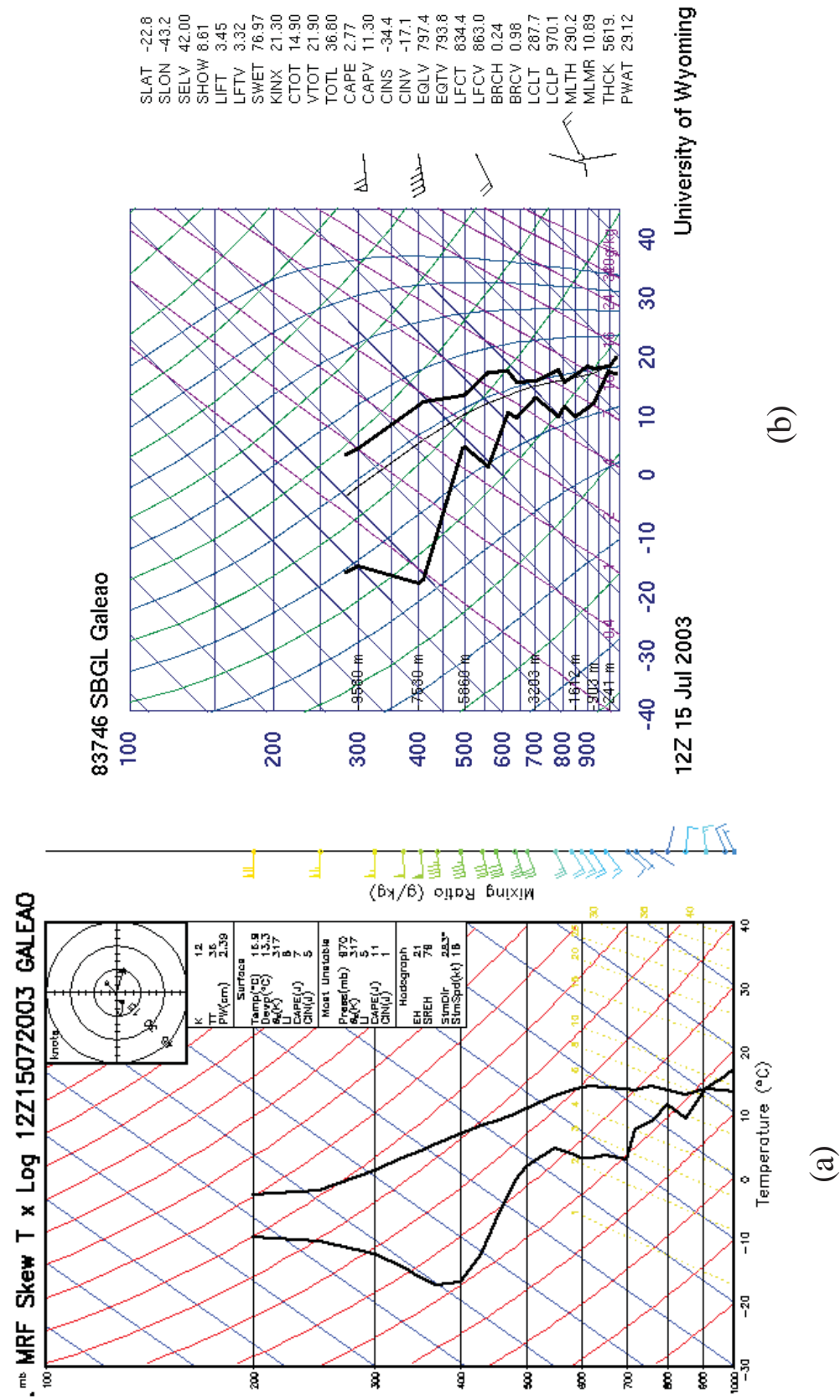

Figura 11 Sondagem referente ao dia 15 de julho de 2003 as 12Z.(a) MM5; (b) SBGL (Fonte: weather.uwyo.edu/upperair/samer.html) 

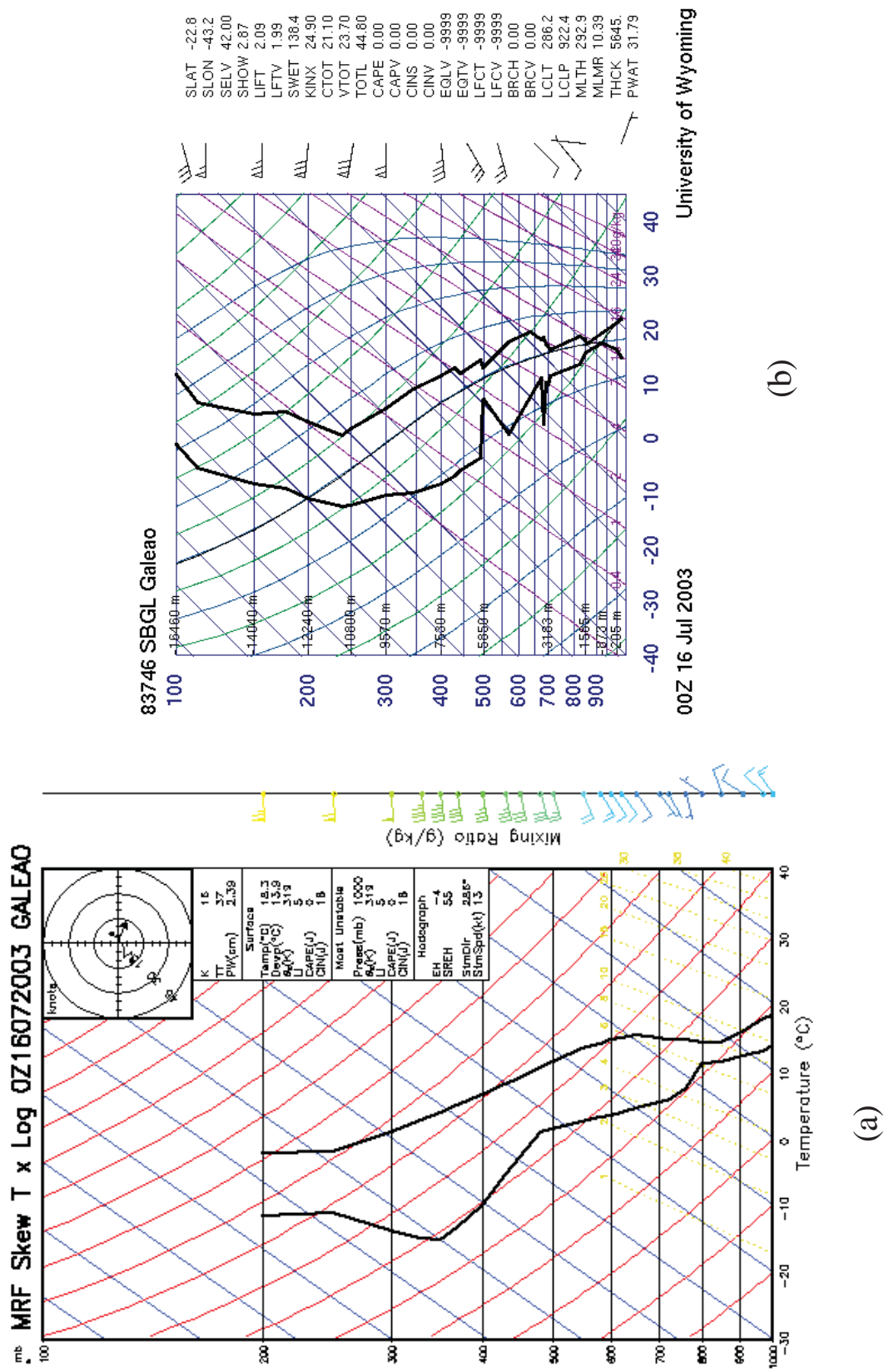

Figura 12 Sondagem referente ao dia 16 de julho de 2003 as 00Z.(a) MM5 ; (b) SBGL (Fonte: weather.uwyo.edu/upperair/samer.html) 
Na Tabela 4 apresentamos os índices estatísticos calculados para o período de 13 a 15 de julho de 2003. O índice EQMR mostra que as simulações realizadas com o modelo MM5 obtiveram melhor desempenho nas faixas de 1 a $3 \mathrm{~m} / \mathrm{s}$ e de 3 a $5 \mathrm{~m} / \mathrm{s}$, o que foi coerente com o indicado pelos índices $\mathrm{Fa} 2$ e Fb e Fs, que demonstram que nessas faixas os resultados obtidos com o modelo MM5 apresentaram menor subestimativa que os valores de intensidade do vento. O coeficiente de correlação apresentou valores baixos e negativos para esse período. A análise de todo o período demonstra o comportamento discutido anteriormente onde os resultados obtidos com o modelo, com essa configuração, apresentam uma tendência a subestimar as intensidades de vento.

\begin{tabular}{|c|c|c|c|c|c|c|c|}
\hline $\begin{array}{c}\text { Qtd } \\
\text { elementos }\end{array}$ & $\begin{array}{c}\text { Faixas de } \\
\text { Intensidade (m/s) }\end{array}$ & EQMA (m/s) & EQMR (\%) & COR & FA2 & FB & FS \\
\hline $15(10)^{*}$ & $0 \leq 1 \leq 1$ & 0.8 & 78 & 0.1 & 1.7 & -0.5 & -2.0 \\
\hline 38 & $1<1 \leq 3$ & 0.8 & 41 & 0.1 & 0.9 & 0.1 & -0.5 \\
\hline 11 & $3<1 \leq 5$ & 1.7 & 41 & 0 & 0.6 & 0.5 & -0.6 \\
\hline 8 & $5<1$ & 4.8 & 70 & -0.5 & 0.3 & 1.1 & 0.6 \\
\hline 72 & 4 dias & 1.5 & 50 & 0.1 & 0.7 & 0.4 & 0.7 \\
\hline
\end{tabular}

Tabela 4 Indicadores estatísticos por faixas de intensidade de vento para o período de 13 a 15 de julho de 2003. Simulações com MM5

\section{Conclusões}

Neste trabalho foram realizadas diversas simulações com o modelo de mesoescala MM5 com o objetivo de analisar sua capacidade no prognóstico dos campos de vento, temperatura e umidade relativa do ar em diversos níveis da atmosfera na RMRJ. Foi escolhida para esse trabalho a parametrização MRF disponível no MM5 para a solução dos fluxos turbulentos.

Os períodos escolhidos foram os dias 10 a 12 de julho de 2003, onde ocorreu a passagem de um sistema frontal pelo estado do Rio de Janeiro e os dias 13 a 15 de julho de 2003 quando o estado estava sob influência de um sistema de alta pressão.

Em relação ao período de 10 a 12 de julho as simulações realizadas com o modelo MM5 demonstraram razoável capacidade em acompanhar as 


\section{Análise Comparativa das Simulações do Modelo de Mesoescala MM5 e Dados Meteorológicos Observados para a Região do Galeão/RJ \\ Eduardo Barbosa Corrêa; Márcio Cataldi \& Luiz Cláudio Gomes Pimentel}

direções predominantes do campo de vento a $10 \mathrm{~m}$. Os resultados obtidos demonstraram uma tendência a subestimar a intensidade do vento, que, aparentemente torna-se mais evidente conforme essa intensidade aumenta. As simulações do modelo MM5 apresentaram melhores resultados para os prognósticos de vento na faixa de intensidade de 1 a $3 \mathrm{~m} / \mathrm{s}$. O vento em médios e altos níveis da atmosfera foi simulado adequadamente.

Quanto aos perfis verticais atmosféricos, as simulações realizadas demonstraram capacidade em capturar o comportamento das curvas de $\mathrm{T}$ e $\mathrm{Td}$. Os perfis verticais de temperatura foram bem representados pelas simulações realizadas com o modelo MM5 durante o período de simulação.

Em relação ao período de 13 a 15 de julho as simulações realizadas representaram adequadamente as direções do campo de vento a $10 \mathrm{~m}$, apesar de permanecer a tendência dos prognósticos do modelo em subestimar sua intensidade. Neste período as simulações realizadas com o modelo MM5 obtiveram melhor desempenho na faixa de intensidade de 1 a $3 \mathrm{~m} / \mathrm{s}$. O campo de vento em médios e altos níveis foi simulado corretamente. Os perfis verticais de Td simulados apresentaram maior concordância com os dados observados nos primeiros níveis da atmosfera. Os resultados obtidos com o modelo apresentaram uma tendência a superestimar a umidade em altos níveis.

Vale ressaltar que os resultados das simulações apresentados neste trabalho foram extraídos de uma célula da grade que possui 3 x $3 \mathrm{~km}$, onde um campo de vento médio é calculado pelo modelo para toda essa área. Este resultado está sendo comparado com um único ponto, sendo que quanto maior a área do ponto de grade, maior será a tendência do modelo em amortecer os picos de maior intensidade. Essa configuração de domínios foi escolhida em virtude do custo computacional requerido para a inclusão de mais um domínio aninhado com uma maior resolução horizontal.

Os resultados deste trabalho apontaram alguns padrões de comportamentos do modelo para as situações estudadas, compatíveis com resultados já verificados anteriormente por Costa e Souza (2002), Atmet (2002), Arellano et alii (2002) e Bright \& Mullen (2002).

Pretende-se dar continuidade a este trabalho utilizando malhas mais refinadas e estendendo as simulações para um período representativo da estação de verão no Rio de Janeiro, incorporando outras parametrizações de Camada Limite Atmosférica disponíveis no modelo MM5. Além disso, também serão estudados os outros modelos paramétricos de solo do MM5, que podem trazer benefícios à simulação dos campos de temperatura e umidade. 


\section{Agradecimentos}

Os autores agradecem ao CNPq e FAPERJ pelo apoio dado no desenvolvimento do trabalho através do Programa de Apoio a Núcleos de Excelência intitulado Núcleo de Excelência em Turbulência.

\section{Bibliografia}

Arellano, J.; Vellinga, O. S.; Holtlang, A.A.M.; Bosveld, F.C. \& Baltink, H. K. 2002. Observational Evaluation of PBL Parametrizations modelled by MM5. In: The Eleventh PSU/NCAR Mesoscale Model Users’ Workshop, 25-27 June 2001, Boulder, Colorado. Disponível em www.mmm.ucar.edu/ $\mathrm{mm} 5$ /papers.html

ATMET. 2002. Highresolution(1.33 km)MM5modeling of the September 1993COAST episode: Sensitivity to model configuration and performance optimization. Report TNRCC 582-0-31984, Report Atmet LLC, Boulder, Colorado, 62 p.

Bright, D. \& Mullen, S. 2002. The sensitivity of the numerical simulation of the southwest monsonn boundary layer to the choice of PBL turbulence parametrization. Weather and Forecasting, 17: 99-114.

Corrêa E. B. Análise da Sensibilidade do Modelo MM5 aos Refinamentos e Aninhamentos de Grades - Um Estudo de Caso Sobre a Cidade do Rio de Janeiro, In: XIII CONGRESSO BRASILEIRO DE METEOROLOGIA, Fortaleza, 2004

Costa e Souza, T., A., S., 2002. Previsão Meteorológica em Portugal Continental Utilizando o Modelo Operacional e de Investigação MM5, M. Sc. Universidade Técnica de Lisboa Instituto Superior Técnico, Lisboa, Portugal , Dissertação de Mestrado, 120p

Dudhia, J., 1989: "Numerical study of convection observed during the winter monsoon experiment using a mesoscale two-dimensional model.” $J$. Atmos. Sci., v. 46, pp. 3077-3107

Dudhia, J. 1996. A multi-layer soil temperature model for MM5. In: Preprint from the Sixth PSU/NCAR Mesoscale Model Users' Workshop. Disponível em www.mmm.ucar.edu/mm5/papers.html

Dudhia, J.; Gill, D.; Guo, Y.; Manning, K.; Wang, W. \& Chiszar, J. 2001: Mesoscale Modeling System Tutorial Class Notes and User's Guide: MM5 Modeling System Version 3, PSU/NCAR.

Grell, G.1993: Prognostic evaluation of assumptions used by cumulus parameterizations. Monthey Weather Review, 121:764-787.

Grell, G. A., Dudhia, J., \& Stauffer, D. R., 1995. "A description of the fifthgeneration Penn State/ NCAR mesoscale model (MM5).” NCAR Technical Note, NCAR/TN-398+STR, 117 p.

Hong, S.-Y., \& Pan, H.-L. 1996. Nonlocal boundary layervertical diffusion in a mediumrange forecast model. Monthey Weather Review, 124: 2322-2339.

Troen, I. \& Mahrt, L., 1986: A Simple Model of the Atmospheric Boundary Layer: Sensivity to Surface Evaporation, Boundary Layer Meteorology, 37:129-148 